\title{
Improved Power for Characterizing Longitudinal Amyloid- $\beta$ PET Changes and Evaluating Amyloid-Modifying Treatments with a Cerebral White Matter Reference Region
}

\author{
Kewei Chen ${ }^{1-4}$, Auttawut Roontiva ${ }^{1,4}$, Pradeep Thiyyagura ${ }^{1,4}$, Wendy Lee ${ }^{1,4}$, Xiaofen Liu ${ }^{1,4}$, Napatkamon Ayutyanont ${ }^{1,4}$, \\ Hillary Protas ${ }^{1,4}$, Ji.Luo Luo ${ }^{1,4}$, Robert Bauer ${ }^{1,4}$, Cole Reschke ${ }^{1,4}$, Daniel Bandy ${ }^{1,4}$, Robert A. Koeppe ${ }^{5}$, Adam S. Fleisher ${ }^{4,6,7}$, \\ Richard J. Caselli ${ }^{4,8}$, Susan Landau ${ }^{9}$, William J. Jagust ${ }^{9}$, Michael W. Weiner ${ }^{10-12}$, and Eric M. Reiman ${ }^{1,4,13,14}$, for the \\ Alzheimer's Disease Neuroimaging Initiative

\begin{abstract}
${ }^{1}$ Banner Alzheimer's Institute, Phoenix, Arizona; ${ }^{2}$ Department of Mathematics and Statistics, Arizona State University, Tempe, Arizona; ${ }^{3}$ Department of Neurology, College of Medicine, University of Arizona, Phoenix, Arizona; ${ }^{4}$ Arizona Alzheimer's Consortium, Phoenix, Arizona; ${ }^{5}$ Division of Nuclear Medicine, Department of Radiology, University of Michigan, Ann Arbor, Michigan; ${ }^{6}$ Eli Lilly and Company, Indianapolis, Indiana; ${ }^{7}$ Department of Neuroscience, University of California-San Diego, San Diego, California; ${ }^{8}$ Mayo Clinic, Scottsdale, Arizona; ${ }^{9}$ School of Public Health and Helen Wills Neuroscience Institute, University of CaliforniaBerkeley, Berkeley, California; ${ }^{10}$ Department of Radiology, University of California-San Francisco, San Francisco, California; ${ }^{11}$ Department of Medicine, University of California-San Francisco, San Francisco, California; ${ }^{12}$ Department of Psychiatry, University of California-San Francisco, San Francisco, California: ${ }^{13}$ Division of Neurogenomics, Translational Genomics Research Institute, Phoenix, Arizona; and ${ }^{14}$ Department of Psychiatry, University of Arizona, Tucson, Arizona
\end{abstract}

In this article, we describe an image analysis strategy with improved power for tracking longitudinal amyloid- $\beta(A \beta)$ PET changes and evaluating $A \beta$-modifying treatments. Methods: Our aims were to compare the power of template-based cerebellar, pontine, and cerebral white matter reference regions to track 24-mo florbetapir standardized uptake value (SUV) ratio (SUVR) changes; to relate those changes to 24-mo clinical declines; and to evaluate $A \beta$-modifying treatments in $A \beta$-positive $(A \beta+)$ and $A \beta$-negative $(A \beta-)$ patients with probable Alzheimer dementia ( $P A D)$, in patients with mild cognitive impairment (MCl), in cognitively normal controls (NCs), and in cognitively normal apolipoprotein E4 (APOE4) carriers and noncarriers. We used baseline and follow-up ( 24 mo) florbetapir PET scans from $332 A \beta+$ and $A \beta$ - subjects participating in the multicenter Alzheimer's Disease Neuroimaging Initiative. Each of the proposed analyses included $31 \mathrm{pAD}$ patients, $187 \mathrm{MCl}$ patients, and $114 \mathrm{NCs}$. Cerebral-to-white matter, cerebellar, and pontine SUVRs were characterized in terms of their longitudinal variability; their power to track longitudinal fibrillar $A \beta$ increases in $A \beta+$ and $A \beta$ - subgroups and cognitively normal APOE4 carriers and noncarriers; the sample sizes needed to detect attenuated accumulation of or clearance of fibrillar $A \beta$ accumulation in randomized clinical trials; and their ability to relate 24-mo fibrillar $A \beta$ increases to clinical declines. Results: As predicted, cerebral-to-white matter SUVR changes were significantly less variable and had significantly greater power to detect 24-mo fibrillar $A \beta$ increases and evaluate $A \beta$-modifying treatment effects in $A \beta+p A D, M C l$, and $\mathrm{NC}$ subjects and cognitively normal APOE4 carriers. They were also distinguished by the ability to detect significant associations between 24-mo $A \beta$ increases and clinical declines. Conclusion: A cerebral white matter reference region may improve the power to track longitudinal fibrillar $A \beta$

Received Oct. 22, 2014; revision accepted Dec. 26, 2014.

For correspondence or reprints contact: Kewei Chen, Banner Alzheimer's Institute and Banner Good Samaritan PET Center, 901 E. Willetta St., Phoenix, AZ 85006.

E-mail: kewei.chen@bannerhealth.com

Published online Mar. 5, 2015.

COPYRIGHT (c) 2015 by the Society of Nuclear Medicine and Molecular Imaging, Inc. increases, to characterize their relationship to longitudinal clinical declines, and to evaluate $A \beta$-modifying treatments in randomized clinical trials.

Key Words: Alzheimer disease; florbetapir PET; biomarkers; image analysis; statistical power; clinical trial sample size

J Nucl Med 2015; 56:560-566

DOI: 10.2967/jnumed.114.149732

$\mathbf{P}$ ET ligands (1-15) have made it possible to investigate the fibrillar amyloid- $\beta$ (A $\beta)$ burden in living people; to clarify its relationship to the dementia, mild cognitive impairment (MCI), and preclinical stages of Alzheimer disease (AD); to characterize the extent to which cross-sectional measurements predict subsequent clinical declines; to track longitudinal changes; and to help evaluate $A \beta$-modifying treatments.

Researchers commonly compute a semiquantitative cerebral-toreference region standardized uptake value ratio (SUVR) using PET counts from a cerebral region of interest (ROI) and from a whole cerebellar $(2,3)$, cerebellar gray matter $(10)$, or pontine $(8)$ reference ROI that is thought to be relatively devoid of fibrillar A $\beta$. Less commonly, researchers compute a cerebral-to-reference region distribution volume ratio or other quantitative measures using longer, dynamically acquired PET scans and the same cerebral and reference ROIs. With conventional reference ROIs, several radioligands have demonstrated a close association between cross-sectional PET SUVR or distribution volume ratio measurements and subsequent postmortem histopathologic measurements of fibrillar $A \beta$ burden and have been used to define relevant thresholds for distinguishing between individuals with and individuals without moderate to severe neuritic plaques $(2,8,16,17)$.

Although longitudinal analyses have the potential to track the progression of fibrillar $\mathrm{A} \beta$ deposition over time, to relate this progression 
to progressive clinical decline, and to evaluate putative $A \beta$-modifying treatments in therapeutic trials, we and others (18-22) have noted substantial variability in longitudinal florbetapir PET measurements of changes in fibrillar $A \beta$ deposition. Some of this variability appears to exceed that expected on the basis of biologic grounds alone (e.g., SUVR changes of $>50 \%$, in either direction, over a matter of weeks).

Among other possible causes, we wondered whether some of the variability in longitudinal A $\beta$ PET measurements might be due to between-session differences in the level or angle of a person's head in the PET scanner and its differential effects on measurements from the cerebral and more inferior cerebellar or pontine ROIs. For instance, if a person's head is positioned a shorter distance into the scanner, then the cerebellum and pons are closer to the inferior limit of the field of view. The resulting reference ROI measurements could be noisier because the 3-dimensional reconstruction algorithm would depend on fewer coincidence events; they could also be preferentially affected by scattered radiation from the body or differentially affected by the way in which the data are reconstructed and corrected for attenuation and scatter. Thus, the use of cerebral and reference ROIs located approximately the same in the axial field of view to generate SUVRs could result in reduced variability in longitudinal SUVRs and improve the power to evaluate $A \beta$-modifying treatments in therapeutic trials. Although we have not yet attempted to clarify whether between-scan differences in scanner head positioning account for the variability in longitudinal florbetapir SUVRs, we were motivated to examine whether a cerebral white matter ROI could reduce the variability in longitudinal florbetapir SUVRs.

In this article, we describe the use of a cerebral white matter reference ROI to reduce the variability in longitudinal florbetapir SUVRs, to improve the sensitivity for detecting corresponding increases, and to improve the power for evaluating $A \beta$-modifying treatments - an approach that is also being evaluated by other groups (18-22). Although cross-sectional cerebral-to-cerebral white matter SUVRs could be affected by the combined effects of fibrillar $A \beta$ and partial-volume averaging, we surmised that this confound could be at least partly reduced in the assessment of longitudinal data because between-session differences in white matter measurements (including those related to the combined effects of small gray matter A $\beta$ PET changes and partialvolume averaging) would be relatively small.

In this study, baseline and 24-mo florbetapir PET data from the Alzheimer's Disease Neuroimaging Initiative (ADNI) were used to determine the extent to which the use of a cerebral white matter reference ROI could reduce longitudinal SUVR variability and improve the power to detect longitudinal increases in fibrillar $A \beta$; to relate longitudinal $A \beta$ deposition and clinical progression; and to estimate the sample sizes needed for the evaluation of fibrillar $A \beta$-modifying treatments in clinical trials. (In the accompanying report, Landau et al. used baseline cerebrospinal fluid $A \beta_{1-42}$ levels and longitudinal florbetapir PET data from the ADNI to demonstrate that a cerebral white matter reference ROI was more accurate than a cerebellar ROI in terms of its accuracy in distinguishing subjects who were expected to have longitudinal SUVR increases from those who were expected to have SUVRs that remained stable over time (22). Their data support the use of a white matter reference ROI in the analysis of longitudinal florbetapir PET scans.)

\section{MATERIALS AND METHODS}

The ADNI is a longitudinal multicenter study that provides a centralized resource for longitudinal clinical, brain imaging, and fluid biomarker data from patients with probable Alzheimer dementia (pAD), patients with MCI, and cognitively normal controls (NCs). The ADNI was launched in 2003 by the National Institute on Aging, the National Institute of Biomedical Imaging and Bioengineering, the Food and Drug Administration, private pharmaceutical companies, and nonprofit organizations. The ADNI was followed by the ADNI-GO and the ADNI-2. We referred to them collectively as ADNI in this study.

This ADNI study was approved by the institutional review boards of the participating institutions. Signed informed written consent was obtained from all participants. To date, over 1,500 adults, 55 to 90 years old, have participated in the ADNI. Current information can be found at http://www.adni-info.org/. For this study, the most fully processed PET data were downloaded from the repository for 332 participants (31 with pAD, 187 with MCI, and 114 NCs) who had undergone florbetapir PET scans at the baseline and at follow-up ( 24 mo) (between May 2010 and December 2013). For our analyses, each subject group was further stratified into $A \beta$-positive $(A \beta+)$ and $A \beta$-negative $(A \beta-)$ subgroups. Twenty-five pAD patients, 73 MCI patients, and $26 \mathrm{NCs}$ were determined to be $\mathrm{A} \beta+$ at the time of the baseline scan with a cerebral-to-whole cerebellar florbetapir SUVR and a threshold of 1.18, which was originally found to distinguish between people with and people without subsequent postmortem evidence of moderate to frequent neuritic plaques. Six $\mathrm{pAD}$ patients, $114 \mathrm{MCI}$ patients, and $88 \mathrm{NCs}$ were determined to be $\mathrm{A} \beta-$ $(2,3)$. The NCs were also stratified into apolipoprotein E4 (APOE4) carrier $(n=30)$ and noncarrier $(n=84)$ subgroups irrespective of their $\mathrm{A} \beta$ status.

The Statistical Parametric Mapping software platform (SPM8; http:// www.fil.ion.ucl.ac.uk/spm/), the FreeSurfer package (https://surfer.nmr. mgh.harvard.edu/), in-house MATLAB (The MathWorks, Inc.) scripts, and step-by-step quality control procedures were used to align each person's 24-mo PET scan with his or her baseline scan; to deform the images into the coordinates of the Montreal Neurologic Institute template; to use the template-based ROIs (independent of each person's own MR imaging) to characterize cerebral target ROI and cerebellar, pontine, and cerebral white matter reference ROI measurements from each scan; and to compute cerebral-to-respective reference region SUVRs.

Cerebral, cerebellar, and pontine ROIs were computed as previously described $(2,3)$. The cerebral white matter ROI was a collection of voxels in the corpus callosum and centrum semiovale, other than those closest to gray matter or to ventricles. The template-based corpus callosum mask was from the WFU_PickAtlas toolbox (http://fmri.wfubmc. edu/software/PickAtlas). The template-based centrum semiovale mask was characterized in our laboratory with FreeSurfer to generate individual MR imaging-based ROIs in 40 randomly selected NCs, to deform the ROIs into Montreal Neurologic Institute coordinates and average them, and to include voxels with a value of at least 0.5 . The templatebased cerebral, cerebellar, pontine, and cerebral white matter ROIs are shown in Figure 1.

For each $\mathrm{A} \beta+, \mathrm{A} \beta-$, cognitively normal APOE4 carrier, and cognitively normal APOE4 noncarrier group, we characterized 24-mo declines in 3 commonly used clinical ratings: decreases in scores on the Mini-Mental State Examination (MMSE), increases in ratings on the 13-item version of the AD Assessment Scale Cognitive Subscale (ADAS-Cog13), and increases in scores on the Clinical Diagnostic Rating Sum of the Box (CDR-SB). We examined the within-group 24-mo cerebral-to-cerebellar, pontine, and cerebral white matter SUVR increases and such increase differences between respective $\mathrm{A} \beta+$ versus $\mathrm{A} \beta$ - or cognitively normal APOE4 carrier versus noncarrier groups. We characterized relationships between 24-mo SUVR 


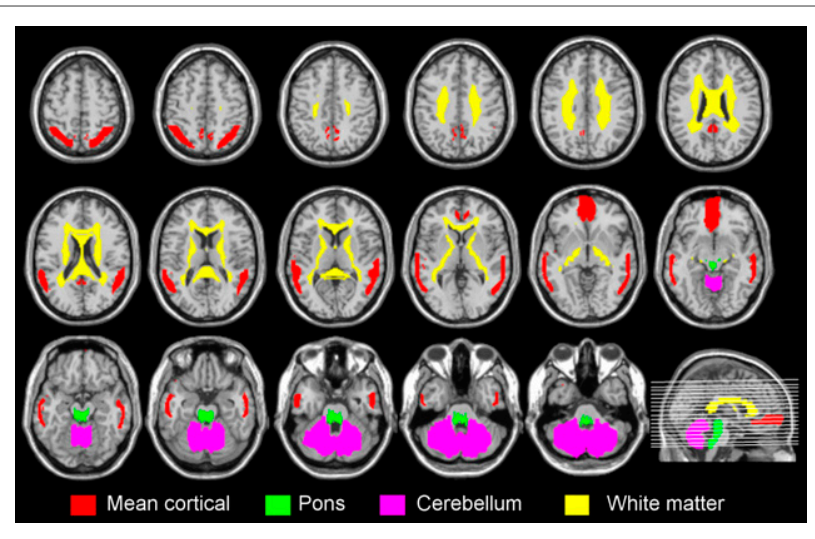

FIGURE 1. Mean cortical (red), cerebellar (magenta), pontine (green), and cerebral white matter (yellow) ROls.

increases and clinical declines using simple Pearson correlation coefficients. $P$ values were computed without correction for multiple comparisons.

For each group, we also estimated the number of participants needed per arm to detect a $25 \%$ effect of treatment in a 12-mo placebo-controlled randomized clinical trial with $80 \%$ power and a 2-tailed $P$ of 0.05 , on the basis of the assumption that SUVR increases were linear. We first estimated the sample sizes needed to detect a $25 \%$ attenuation in further SUVR increases (assuming that the treatments could not remove existing $A \beta$, an assumption that might make sense for the evaluation of a $\beta$-secretase inhibitor). We also estimated the much smaller number of participants needed per arm to detect a $25 \%$ clearance from the baseline level in the treatment group in comparison with the placebo group (an assumption that might make sense for $A \beta$ immunotherapies).

\section{RESULTS}

Demographic characteristics, clinical ratings, and follow-up durations are shown for the $A \beta+$ and $A \beta-$ subgroups in Table 1 and for the cognitively normal APOE4 carrier and noncarrier subgroups in Table 2 .

As shown in Table 3, when the cerebral white matter reference ROI was used, there were small but significant SUVR increases $(P<0.005)$ in the $\mathrm{A} \beta+\mathrm{pAD}$ group, the $\mathrm{A} \beta+$ and $\mathrm{A} \beta-\mathrm{MCI}$ and $\mathrm{NC}$ groups, and the cognitively normal APOE4 carrier and noncarrier groups but not in the $A \beta-$ dementia group. In comparison, when the pontine reference ROI was used, there were small but significant SUVR increases only in the $\mathrm{A} \beta+\mathrm{NC}$ group $(P<0.005)$ and the $\mathrm{A} \beta+\mathrm{MCI}, \mathrm{A} \beta-\mathrm{NC}$, and cognitively normal APOE4 carrier and noncarrier groups $(0.005<P<0.05)$, and there was a failure to detect significant SUVR increases in the $A \beta+$ and $A \beta-$ dementia and $\mathrm{A} \beta-\mathrm{MCI}$ groups. When the cerebellar reference ROI was used, there were small but significant SUVR increases only in the $\mathrm{A} \beta-\mathrm{NC}$ group $(P<0.005)$ and the $\mathrm{A} \beta+$ and cognitively normal APOE4 carrier groups $(0.005<P<0.05)$, and there was a failure to detect significant SUVR increases in any of the dementia or MCI groups. For each $\mathrm{A} \beta+$ or $\mathrm{A} \beta-$ subgroup in the $\mathrm{pAD}, \mathrm{MCI}$, and $\mathrm{NC}$ groups and for each cognitively normal APOE4 carrier or noncarrier subgroup, 24-mo cerebral-to-white matter SUVR increases were significantly greater than cerebral-to-pons SUVR increases $(P<0.05)$, except in the NC A $\beta+$ group $(P=0.065)$. The cerebral-to-white matter SUVR increases over 24 mo were also higher than the cerebral-to-cerebellar SUVR increases in the AD $\mathrm{A} \beta+$ and MCI $\mathrm{A} \beta+$ groups (Table 3 ).

As shown in Figure 2 for individual $A \beta+$ and $A \beta-p A D$ patients together, SUVRs generated with the cerebral white matter ROI appeared less variable and were less likely to show paradoxical decreases than those generated with the pontine and cerebellar ROIs.

TABLE 1

Demographic Characteristics and Clinical Ratings of $A \beta+$ and $A \beta-$ Groups*

\begin{tabular}{|c|c|c|c|c|c|c|c|c|c|}
\hline Characteristic & $\begin{array}{l}\mathrm{AD} \mathrm{A} \beta+ \\
(n=25)\end{array}$ & $\begin{array}{l}\mathrm{AD} A \beta- \\
(n=6)\end{array}$ & $P$ & $\begin{array}{c}\mathrm{MCl} \mathrm{A} \beta+ \\
(n=73)\end{array}$ & $\begin{array}{l}\mathrm{MCl} A \beta- \\
(n=114)\end{array}$ & $P$ & $\begin{array}{l}\mathrm{NCA} \beta+ \\
\left(n=26^{\dagger}\right)\end{array}$ & $\begin{array}{l}\mathrm{NC} \mathrm{A} \beta^{-} \\
\left(n=88^{\ddagger}\right)\end{array}$ & $P$ \\
\hline Baseline age $(\mathrm{y})$ & $76.1 \pm 7.4$ & $81.0 \pm 6.8$ & 0.15 & $74.5 \pm 7.1$ & $70.3 \pm 8.3$ & $<0.001$ & $77.2 \pm 4.6$ & $77.7 \pm 6.5$ & 0.72 \\
\hline Sex (no. of men/women) & $13 / 12$ & $5 / 1$ & 0.16 & $46 / 27$ & $60 / 54$ & 0.16 & $16 / 10$ & $48 / 40$ & 0.53 \\
\hline $\begin{array}{l}\text { No. of subjects with } 0 / 1 / 2 \\
\text { APOE } 4 \text { alleles }\end{array}$ & $3 / 16 / 6$ & $6 / 0 / 0$ & $<0.001$ & $23 / 40 / 10$ & $84 / 27 / 3$ & $<0.001$ & $13 / 12 / 1$ & $71 / 17 / 0$ & 0.003 \\
\hline Education (y) & $15.4 \pm 3.1$ & $16.2 \pm 2.9$ & 0.57 & $16.0 \pm 3.0$ & $16.3 \pm 2.6$ & 0.40 & $16.0 \pm 2.5$ & $16.6 \pm 3.0$ & 0.40 \\
\hline Follow-up duration (mo) & $24.4 \pm 3.2$ & $23.4 \pm 1.1$ & 0.48 & $24.4 \pm 2.3$ & $24.1 \pm 2.3$ & 0.49 & $23.8 \pm 0.6$ & $23.9 \pm 1.8$ & 0.76 \\
\hline Baseline MMSE score & $21.7 \pm 3.4$ & $21.0 \pm 3.6$ & 0.65 & $27.8 \pm 1.8$ & $28.4 \pm 1.6$ & 0.02 & $28.8 \pm 1.2$ & $29.1 \pm 1.3$ & 0.25 \\
\hline $\begin{array}{l}\text { 24-mo MMSE score } \\
\text { decrease }\end{array}$ & $-3.7 \pm 3.1$ & $-1.3 \pm 5.7$ & 0.17 & $-1.2 \pm 2.3$ & $-0.2 \pm 2.2$ & 0.004 & $-0.5 \pm 2.0$ & $-0.4 \pm 1.4$ & 0.67 \\
\hline $\begin{array}{l}\text { Baseline ADAS-Cog13 } \\
\text { rating }\end{array}$ & $31.0 \pm 10.0$ & $30.8 \pm 9.4$ & 0.97 & $16.3 \pm 6.4$ & $11.9 \pm 5.9$ & $<0.001$ & $11.2 \pm 5.7$ & $9.0 \pm 4.2$ & 0.04 \\
\hline $\begin{array}{l}\text { 24-mo ADAS-Cog13 } \\
\text { rating increase }\end{array}$ & $8.2 \pm 6.3$ & $6.3 \pm 10.2$ & 0.56 & $1.8 \pm 5.1$ & $-0.5 \pm 4.3$ & 0.001 & $-0.4 \pm 4.6$ & $0.4 \pm 3.2$ & 0.33 \\
\hline Baseline CDR-SB score & $5.5 \pm 3.3$ & $5.8 \pm 1.3$ & 0.85 & $1.6 \pm 1.0$ & $1.2 \pm 0.8$ & 0.001 & $0.1 \pm 0.4$ & $-.03 \pm 0.7$ & 0.30 \\
\hline $\begin{array}{l}\text { 24-mo CDR-SB score } \\
\text { increase }\end{array}$ & $2.3 \pm 2.2$ & $1.8 \pm 2.1$ & 0.61 & $0.8 \pm 1.6$ & $0.01 \pm 1.4$ & 0.001 & $0.4 \pm 0.8$ & $0.2 \pm 1.1$ & 0.28 \\
\hline
\end{tabular}

${ }^{*}$ Data are reported as mean \pm SD unless otherwise indicated.

${ }^{\dagger}$ Ratings on ADAS-Cog13 were not available for 3 of these subjects.

${ }^{\ddagger_{S}}$ cores on MMSE and ratings on ADAS-Cog13 were not available for 5 and 6 of these subjects, respectively. 
TABLE 2

Demographic Characteristics and Clinical Ratings of Cognitively Normal APOE4 Carrier and Noncarrier Groups

\begin{tabular}{|c|c|c|c|}
\hline \multirow[b]{2}{*}{ Characteristic } & \multicolumn{2}{|c|}{ Mean \pm SD for: } & \multirow[b]{2}{*}{$P$} \\
\hline & APOE4 carriers $\left(n=30^{\star}\right)$ & APOE4 noncarriers $\left(n=84^{\dagger}\right)$ & \\
\hline Baseline age (y) & $77.0 \pm 5.4$ & $77.7 \pm 6.3$ & 0.58 \\
\hline Sex (no. of men/women) & $17 / 13$ & $47 / 37$ & 0.95 \\
\hline Education (y) & $15.9 \pm 3.0$ & $16.6 \pm 2.8$ & 0.25 \\
\hline Follow-up duration (mo) & $24.4 \pm 2.3$ & $23.6 \pm 1.2$ & 0.04 \\
\hline Baseline MMSE score & $28.7 \pm 1.3$ & $29.2 \pm 1.2$ & 0.07 \\
\hline 24-mo MMSE score decrease & $-0.2 \pm 1.8$ & $-0.5 \pm 1.4$ & 0.28 \\
\hline Baseline ADAS-Cog13 rating & $10.9 \pm 4.6$ & $9.0 \pm 4.6$ & 0.07 \\
\hline 24-mo ADAS-Cog13 rating increase & $0.7 \pm 4.4$ & $-0.02 \pm 3.2$ & 0.33 \\
\hline Baseline CDR-SB score & $0.1 \pm 0.4$ & $-0.03 \pm 0.7$ & 0.32 \\
\hline 24-mo CDR-SB score increase & $0.1 \pm 0.6$ & $0.3 \pm 1.2$ & 0.56 \\
\hline
\end{tabular}

*Scores on MMSE and ratings on ADAS-Cog13 were not available for 1 and 4 of these subjects, respectively.

tScores on MMSE and ratings on ADAS-Cog13 were not available for 4 and 5 of these subjects, respectively.

The trend toward changes in cerebral-to-cerebral white matter SUVRs from the baseline to the follow-up at 24 mo seemed more consistent across individual patients (and therefore resulted in reduced variability) (Fig. 2). Similar findings were observed in the other groups.

As shown in Table 4, the estimated number of participants needed per arm to detect a $25 \%$ effect of treatment on further SUVR increases in a 12-mo placebo-controlled randomized clinical trial with $80 \%$ power and a 2 -tailed $P$ value of 0.05 was far smaller when the cerebral white matter reference ROI was used than when either the pontine or the cerebellar reference ROI was used, regardless of subject group. Because the power estimates were made for treatments that were not expected to actually clear existing fibrillar $A \beta$, the number of participants needed was quite high; still, the number was dramatically smaller when the cerebral white matter reference ROI was used.

As also shown in Table 4, the estimated number of participants needed to detect a $25 \%$ decrease in the SUVR from the baseline to 12 mo was much smaller than that needed to detect attenuation in further SUVR increases. Still, the number needed to detect this treatment effect was smaller when the cerebral white matter reference ROI was used than when either the pontine or the cerebellar reference ROI was used.

Correlations between 24-mo SUVR increases and clinical declines in the aggregate group of $31 \mathrm{pAD}$ patients, $187 \mathrm{MCI}$ patients, and 114 NCs with the different reference ROIs are shown in Table 5. The cerebral white matter reference ROI permitted us to demonstrate significant correlations between 24-mo SUVR increases and 24-mo MMSE score decreases $(R=-0.22)$, ADAS-Cog13 rating increases $(R=0.24)$, and CDR-SB score increases $(R=$ $0.22)(P<0.001)$. Significant correlations in the predicted direction were not observed with either the pontine or the cerebellar reference ROI. (None was able to detect such significant correlations in the individual subject groups, probably because of limited sample sizes.)

\section{DISCUSSION}

The present study demonstrated the improved power of florbetapir SUVRs to track longitudinal fibrillar $A \beta$ increases, to characterize their relationship to longitudinal clinical declines, and to

TABLE 3

24-Month SUVR Changes with Pontine, Cerebellar, and Cerebral White Matter ROIs*

\begin{tabular}{|c|c|c|c|c|c|c|c|c|c|c|c|c|}
\hline $\mathrm{ROI}$ & $A D A \beta+$ & $A D A \beta-$ & $P^{\dagger}$ & $\mathrm{MCl} \mathrm{A \beta +}$ & $\mathrm{MCl} A \beta-$ & $P^{\dagger}$ & $N C A \beta+$ & $\mathrm{NC} A \beta-$ & $P^{\dagger}$ & $\mathrm{NC} \varepsilon 4+$ & NC ع4- & $P^{\dagger}$ \\
\hline Pons & $-0.000 \pm 0.030$ & $-0.013 \pm 0.017$ & 0.34 & $0.007 \pm 0.028^{\ddagger}$ & $0.000 \pm 0.018$ & 0.02 & $0.014 \pm 0.024^{\S}$ & $0.005 \pm 0.017^{\ddagger}$ & 0.03 & $0.010 \pm 0.019^{\ddagger}$ & $0.006 \pm 0.019^{\ddagger}$ & 0.32 \\
\hline Cerebellum & $0.004 \pm 0.064$ & $0.011 \pm 0.039$ & 0.80 & $0.007 \pm 0.051$ & $0.004 \pm 0.023$ & 0.33 & $0.024 \pm 0.045^{\ddagger}$ & $0.006 \pm 0.022^{\S}$ & 0.006 & $0.018 \pm 0.039^{\ddagger}$ & $0.008 \pm 0.025^{\ddagger}$ & 0.08 \\
\hline $\begin{array}{l}\text { Cerebral } \\
\text { white matter }\end{array}$ & $0.043 \pm 0.037^{\S}$ & $0.019 \pm 0.027$ & 0.15 & $0.024 \pm 0.028^{\S}$ & $0.008 \pm 0.020^{\S}$ & $<0.001$ & $0.026 \pm 0.021^{\S}$ & $0.011 \pm 0.019^{\S}$ & 0.001 & $0.020 \pm 0.02^{\S}$ & $0.012 \pm 0.021^{\S}$ & 0.08 \\
\hline $\begin{array}{l}\text { White matter } \\
\text { vs. pons } \|\end{array}$ & $<0.001$ & 0.04 & & $<0.001$ & 0.003 & & 0.065 & 0.031 & & 0.055 & 0.04 & \\
\hline $\begin{array}{l}\text { White matter } \\
\text { vs. cerebellum }\end{array}$ & 0.01 & 0.69 & & 0.02 & 0.13 & & 0.86 & 0.15 & & 0.85 & 0.19 & \\
\hline
\end{tabular}

${ }^{*}$ Data are reported as mean \pm SD SUVR changes unless otherwise indicated.

${ }^{\dagger} P$ values reflect differences between respective $A \beta+$ vs. A $\beta$ - or NC APOE4 carrier vs. noncarrier SUVR changes.

†For within-group SUVR increases, $P$ value was $>0.005$ but $\leq 0.05$.

${ }^{5}$ For within-group SUVR increases, $P$ value was $\leq 0.005$.

Data are reported as $P$ values, which reflect SUVR change differences between use of cerebral white matter vs. pons or between use of cerebral white matter vs. cerebellum in each group. 


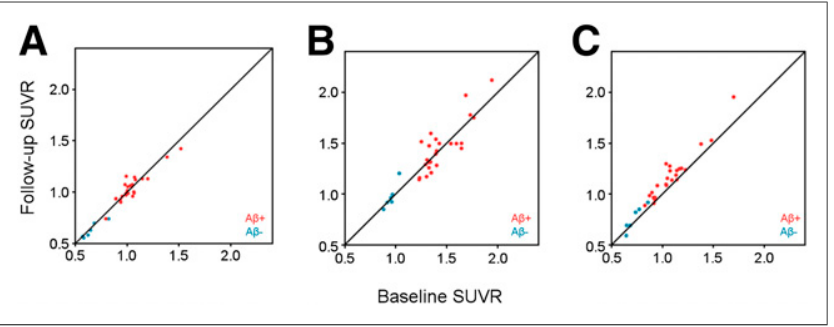

FIGURE 2. Fewer individual variations in tracking of increases in 24mo mean cortical SUVRs with cerebral white matter ROI (C) than with pontine $\mathrm{ROI}(\mathrm{A})$ or cerebellar ROI (B), illustrated for $31 \mathrm{pAD}$ patients. Red and blue dots are data for $A \beta+$ and $A \beta-p A D$ patients.

evaluate $A \beta$-modifying treatments with a cerebral white matter reference ROI instead of a cerebellar or pontine reference ROI.

Our decision to test the ability of the cerebral white matter reference ROI to improve power in longitudinal studies was based on the possibility that some of the variability in sequential SUVRs is attributable to the combined effects of differences in betweenscan head positioning and the differential impact on measurements in a cerebral ROI (near the center of the field of view) and cerebellar and pontine ROIs (closer to the inferior field of view). We reasoned that measurements from a cerebral white matter reference ROI located approximately in the same plane as the cerebral ROI would be less vulnerable to such effects. We further reasoned that PET measurements from the cerebral white matter ROI would be less likely to be confounded by the combined effects of partial-volume averaging and spillover from cerebral gray matter because fibrillar $A \beta$ measurements are unlikely to change over a relatively limited time.

Although our findings support the use of a cerebral white matter reference ROI for the analysis of longitudinal florbetapir PET scans, additional studies are needed to clarify whether the observed improvements in longitudinal tracking are due to differences in head repositioning in PET scanners or to other factors that influence the reference ROI measure. We are now seeking radiation dose and patient body weight data from the ADNI and other studies to compute SUVs and to clarify the extent to which there is a preferential decline in our white matter ROI SUVs (e.g., because of progressive ventricular enlargement), the extent to which there is a preferential decline in gray matter SUVs (e.g., because of progressive gray matter atrophy), and the extent to which the variability in repeated SUVs differs among white matter, cerebellar, and pontine ROIs. We predict that our white matter ROI may lead to a slight overestimation of SUVR increases, particularly in $\mathrm{AD}$ patients, but that this possibility does not solely account for the improvements in the power to track SUVRs. Because $\mathrm{A} \beta$ PET radioligands differ in their nonspecific white matter binding, it remains to be determined whether our findings can be generalized to other radioligands and to more quantitative measurements, such as the distribution volume ratio (23). Although we have not yet clarified whether longitudinal changes in white matter integrity or nonspecific binding could increase variability in cerebral-to-white-matter SUVRs, we were still able to detect improvements in precision between baseline and follow-up SUVRs. Finally, we do not believe that the improvements in SUVR variability were due to the size of the reference ROI and improved counting statistics, because the number of voxels $(10,482)$ in our white matter ROI was smaller than that in the cerebellar ROI $(13,583)$ and because we continued to see improved precision in a post hoc analysis of SUVRs with voxels from only the corpus callosum.

In the present study, we used a template-based cerebral white matter ROI consisting of voxels from the corpus callosum and the centrum semiovale. We have also found similar benefits of the cerebral white matter ROI and similar sample size estimates whether one evaluates changes in absolute SUVRs or the percentage change from baseline. Our data are available at the ADNI Web site. Additional studies are needed to compare our findings with those generated from individual MR imaging-based cerebral white matter ROIs. For now, we recommend using our white matter reference ROI for longitudinal analyses, and we have made the template publicly available on the ADNI website. We also recommend the use of a cerebellar or pontine reference ROI in cross-sectional comparisons (e.g., to determine $A \beta$ positivity or negativity).

Using a method with improved power to track longitudinal SUVR increases, we found a relationship between longitudinal SUVR increases and clinical declines in the aggregate group of pAD, MCI, and NC subjects-a possibly novel finding that may provide additional support for the amyloid hypothesis. As mentioned earlier, the use of cerebral white matter ROIs to measure

TABLE 4

Number of Participants Needed Per Arm to Detect Aß-Modifying Treatment Effect in 12-Month Clinical Trial with $80 \%$ Power and $2-$ Tailed $P$ of 0.05

\begin{tabular}{|c|c|c|c|c|c|c|c|c|c|}
\hline $\begin{array}{l}\text { No. of participants } \\
\text { needed to detect... }\end{array}$ & Reference ROI & $A D A \beta+$ & $A D A \beta-$ & $\mathrm{MCl} A \beta+$ & $\mathrm{MCl} A \beta-$ & $N C A \beta+$ & $N C A \beta^{-}$ & $\mathrm{NC} \varepsilon 4+$ & NC ع4- \\
\hline \multirow[t]{3}{*}{$\begin{array}{l}25 \% \text { attenuation in } \\
\text { further SUVR increases }\end{array}$} & $\begin{array}{l}\text { Cerebral white } \\
\text { matter }\end{array}$ & 187 & 515 & 325 & 1,547 & 162 & 819 & 252 & 770 \\
\hline & Cerebellum & 62,809 & 3,040 & 8,076 & 10,844 & 853 & 2,938 & 1,180 & 2,453 \\
\hline & Pons & N/A & $\mathrm{N} / \mathrm{A}$ & 2,718 & 724,200 & 697 & 3,318 & 907 & 2,519 \\
\hline \multirow[t]{3}{*}{$\begin{array}{l}25 \% \text { decrease in } \\
\text { SUVR from baseline }\end{array}$} & $\begin{array}{l}\text { Cerebral white } \\
\text { matter }\end{array}$ & 8 & 21 & 13 & 62 & 7 & 33 & 11 & 31 \\
\hline & Cerebellum & 2,513 & 122 & 324 & 434 & 35 & 118 & 48 & 99 \\
\hline & Pons & $\mathrm{N} / \mathrm{A}$ & $\mathrm{N} / \mathrm{A}$ & 109 & 28,968 & 28 & 133 & 37 & 101 \\
\hline
\end{tabular}

$\mathrm{N} / \mathrm{A}=$ not applicable because SUVR was decreasing with this reference ROI. 
TABLE 5

Correlations Between 24-Month SUVR Increases and Clinical Declines in All Subjects

\begin{tabular}{llccc}
\hline Test & Statistic & Pons & Cerebellum & $\begin{array}{c}\text { Cerebral } \\
\text { white matter }\end{array}$ \\
\hline MMSE & $P$ & 0.80 & 0.36 & $6 e^{-5 \star}$ \\
\hline ADAS-Cog13 & $P$ & 0.01 & 0.05 & -0.22 \\
& $R$ & -0.04 & -0.04 & 0.24 \\
CDR-SB & $P$ & 0.20 & $0.02^{\dagger}$ & $5 e^{-5 *}$ \\
& $P$ & -0.07 & -0.13 & 0.22 \\
\hline
\end{tabular}

*Significant correlation in postulated direction.

${ }^{\dagger}$ Significant correlation, but in opposite direction.

SUVR increases could overestimate longitudinal SUVR increases because of the combined effects of brain atrophy-related ventricular enlargement and partial-volume averaging, such that measurements in the reference ROI are progressively reduced. Although we cannot exclude that possibility, the improved power to characterize longitudinal SUVR increases was similar in the pAD, MCI, and NC groups, despite likely differences in ventricular enlargement rates.

As previously stated, longitudinal florbetapir PET SUVRs were more variable than predicted with a cerebellar or pontine reference ROI, and some of this variability may have been related to technical rather than biologic reasons. Because the technical source of variability could affect the power to detect treatment effects in clinical trials, including some that have already been reported, we recommend a reanalysis of data with a cerebral white matter reference ROI to determine whether any of the A $\beta$-modifying treatments now in development may have a greater effect than previously realized.

\section{CONCLUSION}

Our findings support the use of a cerebral white matter reference ROI to track longitudinal fibrillar $A \beta$ increases with semiquantitative florbetapir PET measurements, to relate those increases to longitudinal clinical declines, and to evaluate $A \beta$-modifying effects with increased statistical power.

\section{DISCLOSURE}

The costs of publication of this article were defrayed in part by the payment of page charges. Therefore, and solely to indicate this fact, this article is hereby marked "advertisement" in accordance with 18 USC section 1734. This study was supported by National Institutes of Health grant UF1AG046150, National Institute on Aging grants R01 AG031581 and P30 AG19610, and the state of Arizona (all to Eric M. Reiman). Data collection and sharing were funded by the ADNI (U01 AG024904) and DOD ADNI (W81XWH-12-2-0012) (both to Michael W. Weiner, the primary investigator). The ADNI is funded by the National Institute on Aging, by the National Institute of Biomedical Imaging and Bioengineering, and through generous contributions from the following: Alzheimer's Association; Alzheimer's Drug Discovery Foundation; BioClinica, Inc.; Biogen Idec Inc.; Bristol-Myers
Squibb Company; Eisai Inc.; Elan Pharmaceuticals, Inc.; Eli Lilly and Company; F. Hoffmann-La Roche Ltd. and its affiliated company Genentech, Inc.; GE Healthcare; Innogenetics, N.V.; IXICO Ltd.; Janssen Alzheimer Immunotherapy Research \& Development, LLC; Johnson \& Johnson Pharmaceutical Research \& Development LLC; Medpace, Inc.; Merck \& Co., Inc.; Meso Scale Diagnostics, LLC; NeuroRx Research; Novartis Pharmaceuticals Corporation; Pfizer Inc.; Piramal Imaging; Servier; Synarc Inc.; and Takeda Pharmaceutical Company. The Canadian Institutes of Health Research is providing funds to support ADNI clinical sites in Canada. Privatesector contributions are facilitated by the Foundation for the National Institutes of Health (www.fnih.org). The grantee organization is the Northern California Institute for Research and Education, and the study is coordinated by the Alzheimer's Disease Cooperative Study at the University of California-San Diego. ADNI data are disseminated by the Laboratory for Neuro Imaging at the University of Southern California. No other potential conflict of interest relevant to this article was reported.

\section{ACKNOWLEDGMENTS}

We thank Vivek Devadas for technical assistance. Data used in the preparation of this article were obtained from the ADNI (http://adni.loni.usc.edu/). As such, the ADNI investigators contributed to the design and implementation of the ADNI or provided data but did not participate in the analysis or writing of this report. A listing of ADNI investigators can be found at http:// adni.loni.usc.edu/wpcontent/uploads/how_to_apply/ADNI_ Acknowledgment_List.pdf.

\section{REFERENCES}

1. Becker GA, Ichise $\mathrm{M}, \mathrm{Barthel} \mathrm{H}$, et al. PET quantification of ${ }^{18} \mathrm{~F}$-florbetaben binding to $\beta$-amyloid deposits in human brains. J Nucl Med. 2013;54:723731.

2. Clark CM, Pontecorvo MJ, Beach TG, et al. Cerebral PET with florbetapir compared with neuropathology at autopsy for detection of neuritic amyloid-beta plaques: a prospective cohort study. Lancet Neurol. 2012;11:669678.

3. Fleisher AS, Chen K, Liu X, et al. Apolipoprotein E epsilon4 and age effects on florbetapir positron emission tomography in healthy aging and Alzheimer disease. Neurobiol Aging. 2013;34:1-12.

4. Hatashita S, Yamasaki H, Suzuki Y, et al. $\left[{ }^{18} \mathrm{~F}\right]$ flutemetamol amyloid-beta PET imaging compared with $\left[{ }^{11} \mathrm{C}\right] \mathrm{PIB}$ across the spectrum of Alzheimer's disease. Eur J Nucl Med Mol Imaging. 2014;41:290-300.

5. Joshi AD, Pontecorvo MJ, Clark CM, et al. Performance characteristics of amyloid PET with florbetapir F 18 in patients with Alzheimer's disease and cognitively normal subjects. J Nucl Med. 2012;53:378-384.

6. Joshi AD, Pontecorvo MJ, Adler L, et al. Radiation dosimetry of florbetapir F 18. EJNMMI Res. 2014:4:4.

7. Klunk WE, Engler H, Nordberg A, et al. Imaging brain amyloid in Alzheimer's disease with Pittsburgh Compound-B. Ann Neurol. 2004;55: 306-319.

8. Leinonen V, Rinne JO, Wong DF, et al. Diagnostic effectiveness of quantitative $\left[{ }^{18} \mathrm{~F}\right]$ flutemetamol PET imaging for detection of fibrillar amyloid beta using cortical biopsy histopathology as the standard of truth in subjects with idiopathic normal pressure hydrocephalus. Acta Neuropathol Commun. 2014; 2:46.

9. Mathis CA, Bacskai BJ, Kajdasz ST, et al. A lipophilic thioflavin-T derivative for positron emission tomography (PET) imaging of amyloid in brain. Bioorg Med Chem Lett. 2002;12:295-298.

10. Reiman EM, Chen K, Liu X, et al. Fibrillar amyloid-beta burden in cognitively normal people at 3 levels of genetic risk for Alzheimer's disease. Proc Natl Acad Sci USA. 2009;106:6820-6825. 
11. Rowe CC, Ackerman U, Browne W, et al. Imaging of amyloid beta in Alzheimer's disease with ${ }^{18} \mathrm{~F}-\mathrm{BAY} 94-9172$, a novel PET tracer: proof of mechanism. Lancet Neurol. 2008;7:129-135.

12. Vandenberghe R, Van LK, Ivanoiu A, et al. ${ }^{18} \mathrm{~F}$-flutemetamol amyloid imaging in Alzheimer disease and mild cognitive impairment: a phase 2 trial. Ann Neurol. 2010;68:319-329.

13. Villemagne VL, Ong K, Mulligan RS, et al. Amyloid imaging with ${ }^{18}$ F-florbetaben in Alzheimer disease and other dementias. J Nucl Med. 2011;52:12101217.

14. Villemagne VL, Mulligan RS, Pejoska S, et al. Comparison of ${ }^{11} \mathrm{C}-\mathrm{PiB}$ and ${ }^{18} \mathrm{~F}-$ florbetaben for $\mathrm{A} \beta$ imaging in ageing and Alzheimer's disease. Eur J Nucl Med Mol Imaging. 2012;39:983-989.

15. Wang H, Guo X, Jiang S, et al. Automated synthesis of $\left[{ }^{18} \mathrm{~F}\right]$ florbetaben as Alzheimer's disease imaging agent based on a synthesis module system. Appl Radiat Isot. 2013;71:41-46.

16. Carome M, Wolfe S. Florbetapir-PET imaging and postmortem beta-amyloid pathology. JAMA. 2011;305:1857-1858.

17. Dugger BN, Clark CM, Serrano G, et al. Neuropathologic heterogeneity does not impair florbetapir-positron emission tomography postmortem correlates. $\mathrm{J} \mathrm{Neu}$ ropathol Exp Neurol. 2014;73:72-80.
18. Baker S, Marks S, Landau S, et al. Exploring the best methods to detect longitudinal change in amyloid imaging. Paper presented at: Human Amyloid Imaging Conference; January 15-17, 2014; Miami, Florida.

19. Matthews D, Marendic B, Randolph A, et al. Longitudinal amyloid measurement for clinical trials: a new approach to overcome variability. Paper presented at: Human Amyloid Imaging Conference; January 15-17, 2014; Miami, Florida.

20. Seibyl J, Sabri O, Barthel H, et al. Modeling the influence of white matter contamination on detectability of brain amyloid changes in longitudinal studies of Alzheimer's progression: segmentation analyses using the PET $\beta$-amyloid tracer ${ }^{18} \mathrm{~F}$ NAV4694. Paper presented at: Human Amyloid Imaging Conference; January 15-17, 2014; Miami, Florida.

21. Koeppe R. The Centiloid method for quantifying amyloid PET studies: great illuminator or master of illusion. Paper presented at: Human Amyloid Imaging Conference; January 15-17, 2014; Miami, Florida.

22. Landau SM, Fero A, Baker SL, et al. Measurement of longitudinal $\beta$-amyloid change with ${ }^{18} \mathrm{~F}$-florbetapir PET and standardized uptake value ratios. $\mathrm{J} \mathrm{Nucl}$ Med. 2015;56:567-574.

23. van Berckel BN, Ossenkoppele R, Tolboom N, et al. Longitudinal amyloid imaging using ${ }^{11} \mathrm{C}-\mathrm{PiB}$ : methodologic considerations. J Nucl Med. 2013;54: $1570-1576$. 